\title{
Bronchoesophageal fistula secondary to postpneumonectomy like syndrome caused by bilobectomy and postoperative radiation therapy for lung cancer
}

\author{
Hee Yun Seol ${ }^{1}$, Taehwa Kim ${ }^{1}$, Sung Kwang Lee ${ }^{2}$, Joo Hyung Son ${ }^{2}$, Do Hyung Kim ${ }^{2}$ \\ ${ }^{1}$ Department of Internal Medicine, ${ }^{2}$ Department of Thoracic and Cardiovascular Surgery, Pusan National University Yangsan Hospital, Yangsan, \\ Gyoungnam, South Korea \\ Correspondence to: Do Hyung Kim, MD. Department of Thoracic and Cardiovascular Surgery, Pusan National University Yangsan Hospital, Pusan National \\ University College of Medicine, 20, Geumo-ro, Mulgeum-eup, Yangsan-si, Gyeongsangnam-do, 50612, South Korea. Email: yumccs@nate.com.
}

\begin{abstract}
Postpneumonectomy like syndrome is a very rare condition and benign bronchoesophageal fistula (BEF) is also uncommon in the adult. Therefore, the probability of occurrence of BEF secondary to postpneumonectomy like syndrome will be extremely rare, and has not been reported yet in the literature. This is the first case report of postpneumonectomy like syndrome combined BEF after bilobectomy and postoperative radiation therapy for lung cancer.
\end{abstract}

Keywords: Bronchoesophageal fistula (BEF); postoperative complication; lung cancer

Submitted May 22, 2019. Accepted for publication Sep 04, 2019.

doi: 10.21037/atm.2019.09.84

View this article at: http://dx.doi.org/10.21037/atm.2019.09.84

\section{Introduction}

Post pneumonectomy like syndrome is a very rare condition and benign bronchoesophageal fistula (BEF) in the adult is also uncommon $(1,2)$. Given the mechanism of benign BEF, the most common cause of it is bronchial or esophageal inflammation, which is difficult to occur with pressurerelated injuries (i.e., erosion) of the Levin tube, the most common cause of tracheoesophageal fistula (TEF) (3). Here we report the first case of post pneumonectomy like syndrome combined with BEF after bilobectomy and postoperative radiation therapy for lung cancer.

\section{Case presentation}

A 58-year-old female who underwent a right middle and lower lobe bi-lobectomy for stage IIB lung cancer (pT2aN1M0, mucoepidermoid carcinoma, low grade) was admitted for dyspnea. Twelve months ago, she visited the emergency department with massive hemoptysis for three days. Chest computed tomography (CT) showed about 4.3 $\mathrm{cm}$ sized lobulated endobronchial mass arising from right bronchus intermediates, right bronchial artery hypertrophy, and no significant lymphadenopathy. After bronchial artery embolization for control of hemoptysis, bronchoscopy showed obstruction with mass mixed with exudate material protruding from right bronchus intermedius. Histologic finding of forcep biopsy was mixed squamous cell and glandular papilloma. She underwent a right middle and lower lobe bi-lobectomy by thoracotomy. She received postoperative radiation therapy because of the perineural invasion. Four weeks after completion of postoperative radiation therapy (total dose of 54 Gy using mixed technique of $45 \mathrm{~Gy}$ in 18 fractions to mediastinum with three-dimensional conformal radiation therapy and 9 Gy in 12 fractions to tumor bed with intensity modulated radiation therapy), radiation pneumonitis occurred and treated with steroids for 3 months. After 4 months, she suddenly developed dyspnea. Chest CT showed new pulmonary artery thrombosis near the stump area, pericardial effusion, and pneumonia. She was treated with pericardiocentesis, antibiotics, and antithrombotic therapy, and dyspnea improved. However, swallowing difficulty and dyspepsia persisted. Upper gastrointestinal endoscopy was performed, it showed extra-luminal compression of the esophagus 


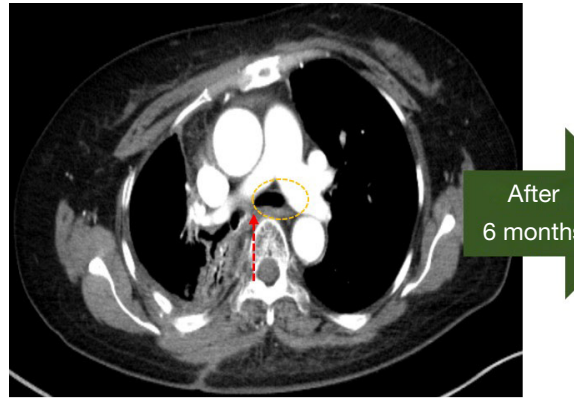

Post operative 1 month

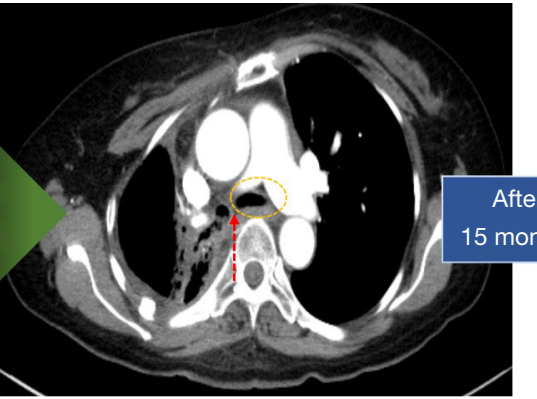

Rt. deviation of mediastinum

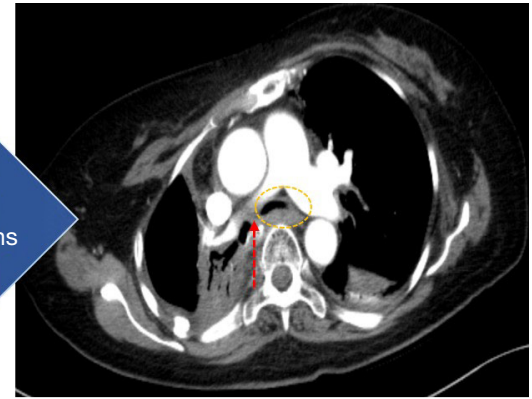

Narrowing of Lt. main bronchus

Figure 1 Serial chest CT shows a gradual decrease in the size of the thoracic cavity with the ongoing right deviation of mediastinum. Red arrow means carina moves to the right side and yellow circle means narrowing of left main bronchus.
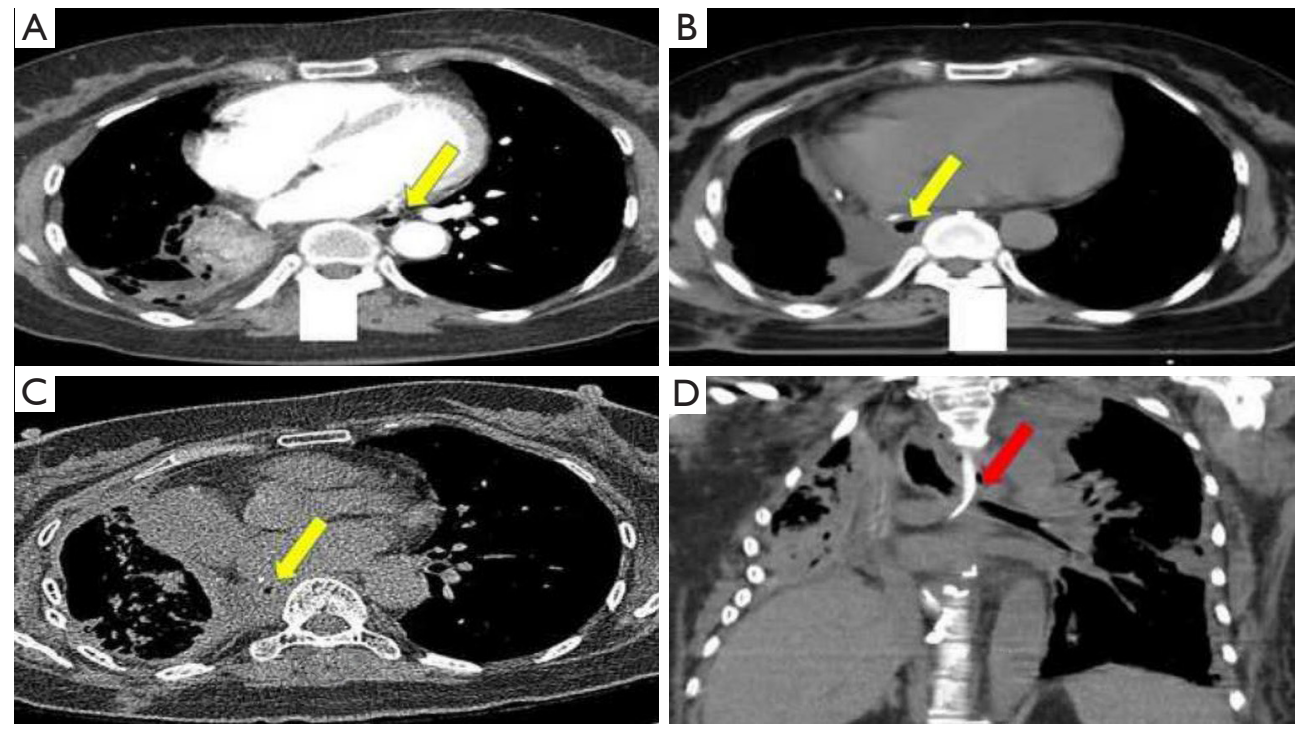

Figure 2 Serial chest CT shows the position of lower esophagus changed from left to the right side after bilobectomy, (A) pre-operation, (B) post operation two months, (C) post-operation 15 months, (D) post-operation 15 months, coronal view, Levin tube in the mid esophagus between left main bronchus and the vertebral body, yellow arrow is esophagus and red arrow is Levin tube.

at 25 to $30 \mathrm{~cm}$ from incisors. After gastro endoscopy, aspiration pneumonitis occurred and mechanical ventilator assist was required. Despite adequate treatment, it had not improved and additional ventilator-induced lung injury has occurred. During ICU care, the size of the thoracic cavity was progressively decreased due to right lung destruction and compensatory expansion of the left lung. During ventilator care, the serial chest CT showed that progressive deviation of the mediastinal structures toward the right side and the lumen of left main bronchus was gradually decreased by extrinsic compression of the left pulmonary artery and vertebral body (Figure 1). And the direction of middle and lower esophagus was changed from left to right side across midline after bilobectomy (Figure $2 A, B, C$ ). This change caused mid esophagus to pass between left main bronchus and the vertebral body under the left main canal (Figure 2D). Serial bronchoscope also shows gradually extrinsic compression was found in the left main bronchus stenosis (Figure 3). Bronchoscopy and CT findings were compatible with post-pneumonectomy like syndrome due to right lung destruction. In last bronchoscope, fungus such as Candida albicans and multidrug resistant bacteria such as Elizabethkingia meningoseptica, and Burkholderia cepacia were identified in the bronchoalveolar lavage. And last chest CT before days extracoporeal membrane oxygenation (ECMO) insertion shows the left main bronchus is compressed by 


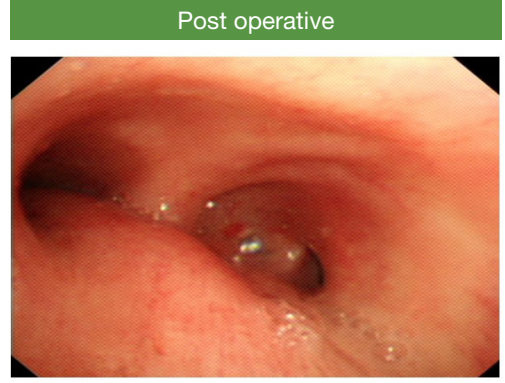

Normal bronchus

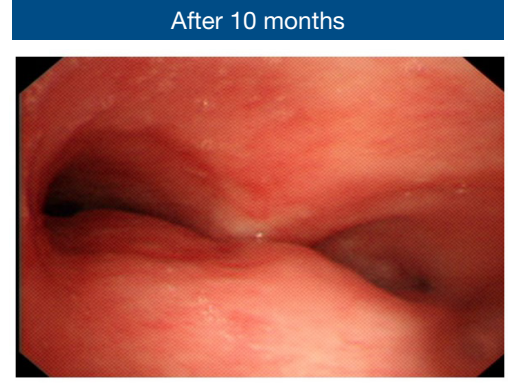

Narrowing of Lt. main bronchus

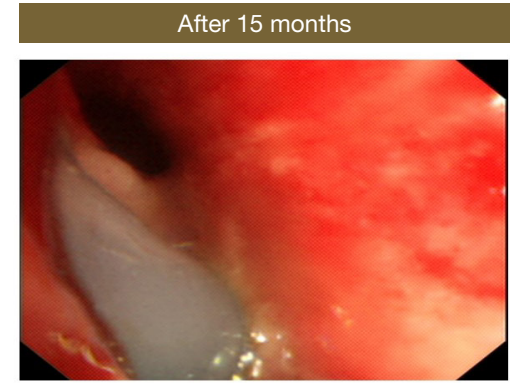

L-tube in the bronchus

Figure 3 Serial bronchoscope also shows gradually extrinsic compression was found in the left main bronchus stenosis. Levin tube was located in the bronchus, suggesting BEF.

the compression between the vertebral body and the right pulmonary artery, resulting in bronchial stenosis and the esophagus is also located behind the left main bronchus (Figure 1).

On post-operation 16 months, 28 days after mechanical ventilation, sudden continuous leakage of air from the Levin tube and a sharp decrease of the tidal volume was developed. After that, it was difficult to maintain respiration even though full ventilator support. ECMO insertion was determined for respiratory function recovery. After ECMO implantation and respiration recovery, bronchoscope performed. On bronchoscopy, the left main bronchus was narrowed due to external compression, and a Levin tube was observed in the bronchus (Figure 3). We planned urgent surgery but acute respiratory distress syndrome deteriorated and the patient died on the 2nd day of ECMO.

\section{Discussion}

Post-pneumonectomy syndrome is a rare pneumonectomy complication which was developed by mediastinum shifts to the site of the removed lung. Mediastinal shifting toward the pneumonectomy space results in bronchial compression between the pulmonary artery, aorta, and vertebral column. Such like phenomenon is not developed only in patients with pneumonectomy, but in case that the lung volume is decreased due to lung destruction, symptoms such as post pneumonectomy syndrome may be seen. Comparing the incidence of post pneumonectomy syndrome is reported to occur in about $0.15 \%$ of pneumonectomy patients, whereas post pneumonectomy like syndrome is rarely reported, there were only less than 10 cases in the world literature. In the reported literature, the cause of lung volume reduction was tuberculosis, chemoradiation treatment, lobectomy, and congenital lung agenesis. Especially, only 3 cases have been reported as surgical complications after lobectomy in the literature (4-6).

The most common cause of tracheoesophageal or bronchoesophageal fistula is malignant tumors around the esophagus and airway. The incidence of benign tracheal or bronchial fistula is very low. Especially, benign BEF is a very rare disease, according to a 2002 Massachusetts General Hospital report, only 13 cases of benign BEF were reported for 40 years (2). Considering the mechanism of $\mathrm{BEF}$ pathophysiology, the most common cause of BEF is bronchial or esophageal inflammation. In most benign TEFs, the Levin tube in esophagus or endotracheal tube (ETT) cuff in trachea pressurize the tissue to make an erosion can cause a fistula. On the other hand, unlike TEF, acquired benign BEF is mainly caused by persistent inflammation of the esophagus or bronchus. Infection of the peri-bronchial or mediastinal nodes leads to enlargement and necrosis of the node, resulting in erosion and rupture of the esophagus and the bronchus, resulting in BEF. BEF is not usually caused by erosional injury by intubation tube or Levin tube like TEF $(2,3)$.

However, we think the mechanism of BEF in this case is pressure-related injuries similar with TEF rather than the typical mechanism of BEF caused by chronic inflammation of esophagus and organ surrounding tissues. This extraordinary mechanism of BEF can be caused by the shifting of mediastinal structure, main bronchus and esophagus by bilobectomy, radiation therapy, and mechanical ventilation. The esophagus was originally located on the left side in the preoperative chest CT but the middle and lower esophagus moved to the right, pneumonectomy side to fill the empty space after operation as shown in the postoperative 1-month chest CT. Therefore, the mid-esophagus 
was located between left main bronchus and vertebral column. Finally, extraordinary BEF was developed due to the pressure-related erosion of esophagus caused by the Levin tube under pressure on both bronchus and vertebral column.

In conclusion, symptoms similar to post-pneumonectomy syndrome may occur in patients with lobectomy under extra-ordinary conditions. When the symptoms are worse, they can be accompanied by BEF. Post pneumonectomy like syndrome in mechanical ventilation can lead to a lethal situation when BEF develops. It always thinks of possibility and requires

\section{Acknowledgments}

None.

\section{Footnote}

Conflicts of Interest: The authors have no conflicts of interest to declare.

Ethical Statement: The authors are accountable for all aspects of the work in ensuring that questions related to the accuracy or integrity of any part of the work are appropriately investigated and resolved. Written informed consent was obtained from the patient for publication of this manuscript and any accompanying images.

\section{References}

1. Lee J, Kim D. Saline prosthesis implantation using an extrapleural approach for the treatment of postpneumonectomy-like syndrome due to tuberculosisdestroyed lung. J Thorac Dis 2016;8:E144-7.

2. Mangi AA, Gaissert HA, Wright CD, et al. Benign broncho-esophageal fistula in the adult. Ann Thorac Surg 2002;73:911-5.

3. Bibas BJ, Cardoso PFG, Minamoto H, et al. Surgery for intrathoracic tracheoesophageal and bronchoesophageal fistula. Ann Transl Med 2018;6:210.

4. Chen F, Takahagi A, Sakamoto K, et al. Lung autotransplantation technique for postpneumonectomylike syndrome. J Thorac Cardiovasc Surg 2015;150:e45-7.

5. Heyndrickx M, Le Rochais JP, Flais F, et al. Postpneumonectomy-like syndrome after lobectomy: An exceptional situation. Asian Cardiovasc Thorac Ann 2015;23:464-6.

6. Stratakos G, Vitsas V, Koufos N, et al. Postpneumonectomy and post-lobectomy syndromes: case series and review of the literature. Monaldi Arch Chest Dis 2017;87:810.
Cite this article as: Seol HY, Kim T, Lee SK, Son JH, Kim DH. Bronchoesophageal fistula secondary to postpneumonectomy like syndrome caused by bilobectomy and postoperative radiation therapy for lung cancer. Ann Transl Med 2019;7(20):588. doi: 10.21037/atm.2019.09.84 